\title{
A Preliminary Investigation into the Usability of Force Touch Interaction in a Mobile Context
}

\author{
https://doi.org/10.3991/ijim.v12i5.9208 \\ Dr Pietro Murano ${ }^{(凶)}$, Rao Muhammad Danial Ali \\ OsloMet - Oslo Metropolitan University, Oslop, Norway \\ pietro.murano@oslomet.no
}

\begin{abstract}
This paper details a preliminary evaluation into the usability and universal design of force touch interaction with older users as recently introduced into the Apple iOS9 operating system. The evaluation involved a small group of older users and two typical smartphone tasks. The tasks involved increasing font size and rotating an image on an Apple iPhone with iOS9 installed. The study recorded task times and semi-structured interview responses. The overall results indicated that the new force touch feature can gain good acceptance from older users, but that there were some usability and universal design problems with the feature which hampered the potential of full acceptance by older users.
\end{abstract}

Keywords-Usability, Evaluation, Force touch, Touch based user interface, Universal design, smartphones.

\section{Introduction}

In recent years touch-based interaction has received a lot of attention from developers. Many every day devices such as telephones, tablets and some laptops use touch screens. Further, some public service type interactive devices also use touch screens. Some examples include ticket vending machines and bank cash machines.

The hardware technology surrounding this area has several underlying designs with four main types of touch screen technology. These are resistive, capacitive, infrared and surface acoustical wave. The touch screen has three main elements, a 'touch sensor, a controller and a software driver'. For a fuller discussion of each the reader is referred to Bhalla and Bhalla's work [1] for a good overview.

Since devices with touch screens inevitably are used by people and many different kinds of user, it is important that the features and interaction capabilities of such touch screen devices are made as usable as possible for everyone with ideally little or no modification required [2].

The interaction options (gestures) a user has with touch screens are varied, e.g. one can use single tap, swipe and pinch interaction mechanisms etc. and recently, Apple introduced force touch in the iOS 9 operating system [3], which is also known as 3DTouch. With force touch, Apple supports 'Quick Actions' and 'Peek and Pop' [3]. Quick Actions allows the system to differentiate and recognise between a very short 
tap and a longer tap. Peek and Pop involves gestures which are sensitive to the amount of pressure a user exerts onto the touch screen.

Linked to the above, the work by the authors of this paper used an Apple iPhone 6s with the aim of investigating the usability of force touch interaction for older users in the context of increasing font size and rotating images. To our knowledge there has not been a systematic usability study of such an interaction mechanism in the context of older users. Furthermore, this initial investigation links in with the universal design principles as detailed in [2].

Therefore, the rest of this paper will cover a number of areas. First, we introduce a brief literature review of related works. Then, a description of the evaluation and results from the evaluation are detailed and finally some conclusions are presented to the reader.

\section{$2 \quad$ Related Literature}

The use of force (force-touch) as a further method or option in an interaction has been around from before the every-day use of touch screens in smartphones and tablets. For example, some researchers have investigated adding sensors underneath a physical mobile telephone keyboard with the idea of detecting pressure [4]. Furthermore, force touch is being considered in contexts beyond smartphones. One example is the idea of using a type of force touch in car steering wheels [5].

Some researchers have concluded that generally touch based user interfaces are better for elderly users. One example is the research by [6]. They conducted a comparison between a touch based user interface and one using a mouse (attached to a laptop). One of their aims was also to compare older users with younger users. Older users in their paper were in the range of 61-86 years of age. Overall the younger participants were faster. However, the speed difference between the older and younger participants was reduced when the touch based user interface was used. Overall error rates were higher under the mouse based tasks. However there were no significant differences in terms of younger or older users for errors.

More study into the possible positive or negative aspects of force touch is useful, because the aim of most Human Computer Interaction (HCI) researchers is to improve user interfaces. Brewster and Hughes [7] carried out some research where they compared two force touch methods (Dwell and Quick Release) for a mobile (tablet) context, in comparison with a 'standard shift key' keyboard. They found that Dwell had the highest accuracy and Quick Release was faster in the context of text being entered that had a mixture of lower case and upper case characters.

Research by Hwangbo, Yoon, Jin, Han and Ji [8] in the context of smartphones and elderly users, sought to find evidence for pointing performance. In this study the authors defined a participant as being elderly if over the age of 65 . The authors' findings indicate that the participants' performance was affected by a target's (e.g. and icon or a keyboard button) characteristics. The size, spacing and target position were influencing factors. Also, the elderly participants performed better with audio tactile feedback. 
In another study comparing thumb-based gestures and finger-based gestures, Kim and Jo [9] found that overall thumb-based gestures were not as efficient as fingerbased gestures. Although their study included in their participant sample individuals over 60 years of age, the authors did not report any specific details in relation to age groups.

Further, Hertzum and Hornbæk [10]conducted a comparative study of pointing with a mouse and a touchpad. This was done with young, adult and elderly users. The authors' elderly group was between 61 and 69 years of age. Their main findings in terms of elderly participants were that they overall were slower than the adult participants. However, the errors made by elderly participants were not any worse than the young or adult groups. The elderly participants were slower with the touchpad when compared to the other age groups.

Concerning universal design, other authors have seen the importance of universal design being linked to user interfaces. For example, Akoumianakis and Stephanidis [11] acknowledged the importance of universal design and HCI.

Story [2] has a good detailed discussion on the seven principles of universal design. The principles (P) are: P1 - Equitable Use, P2 - Flexibility in Use, P3 - Simple and Intuitive Use, P4 - Perceptible Information, P5 - Tolerance for Error, P6 - Low Physical Effort, P7 - Size and Space for Approach and Use. While all the principles are important the work described in this paper will concentrate on the principles that are most relevant for the context of force touch and older users. If force touch is to be 'universal', it suggests that the design of the interaction should be equitable and therefore 'marketable' to people with different skills. This also links with flexibility. Such a design should be flexible enough to allow for different likes and skills. Also, such features should make a design understandable irrespective of the 'kind' of user. The other interesting and relevant principle concerns a design of an artefact successfully communicating to users relevant information for use, e.g. with force touch it should be clear to users that the functionality is available and that it is clear how to use it.

\section{The Evaluation Study With Elderly Participants}

\subsection{Prototype and Tasks}

In this evaluative study the authors wanted to find out if older participants would find using the force touch gesture easier to use than the current conventional options (e.g. pinch-to-zoom and pinch-to-rotate). The context of increasing font size and rotating images was used in this study.

One of the first stages of this evaluative study was to develop a small mobile based prototype for testing and evaluating force touch in the context of typical tasks one might do with a smartphone. This would help to have ecological validity in the evaluation, as atypical tasks would not be realistic. The prototype was developed to act like an interactive web page. The pressure.java script created by Yamartino [12] was used, which has pressure touch building properties to develop a force-to-zoom gesture and force-to-Rotate gesture. 
The prototype was developed to handle four tasks which in turn were carried out by all participants once.

Task 1 involved increasing the text size of web page content to the maximum possible enlargement of $400 \%$. A task was deemed to be completed successfully if the participant was able to increase the text to at least $200 \%$. Users increased the text size with a force gesture (force-to-zoom). For this task a newspaper page was used, as reading the news on a smartphone is a common activity [13]. Figure 1 illustrates Task 1 with dummy text.
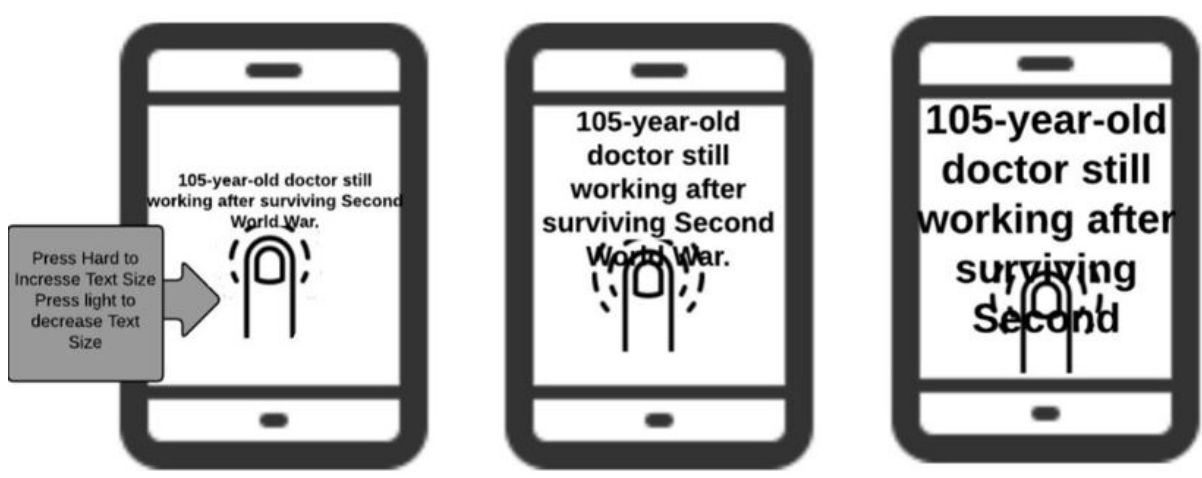

Fig. 1. Task 1 Force-to-zoom

Task 2 was the same as Task 1 but involved carrying it out using the conventional multi-touch gesture (pinch-to-zoom).

Task 3 involved rotating a picture $360^{\circ}$ clockwise using a force touch gesture (force-to-rotate). A task was deemed to be completed successfully if the participant was able to rotate the image at least $180^{\circ}$. The context of manipulating a picture was considered to be a common activity done on a smartphone and therefore valid. The basic data from Statista [13] indicates that many activities done on a smartphone involve highly visual aspects. The picture was placed on a 0 -degree interval around the initial position for this task. The force touch gesture button was able to rotate the picture from $0^{\circ}$ to $360^{\circ}$. In this evaluation, the user was to use the force touch button with one finger. Figure 2 illustrates Task 2.

Task 4 was the same as Task 3 but involved carrying it out by rotating the picture with a multi-touch gesture (pinch-to-rotate).

In practice, the evaluation was carried out on an Apple iPhone 6s smartphone [14]. The size of the screen was 4.7 inches, and it had a resolution of $750 \times 1334$ pixels. This device was chosen because at the time of doing this work it was the only common mobile device which supported force touch (or 3D Touch). It also supported both multi-touch and force touch simultaneously. It had capacitive touch sensing, which supports force gestures. In the evaluation, both touch sensing types were used. Participants were allowed to use any hand (left or right) and any suitable physical position. However, for the force touch tasks participants were instructed to use single fingers while pressing on the screen. 

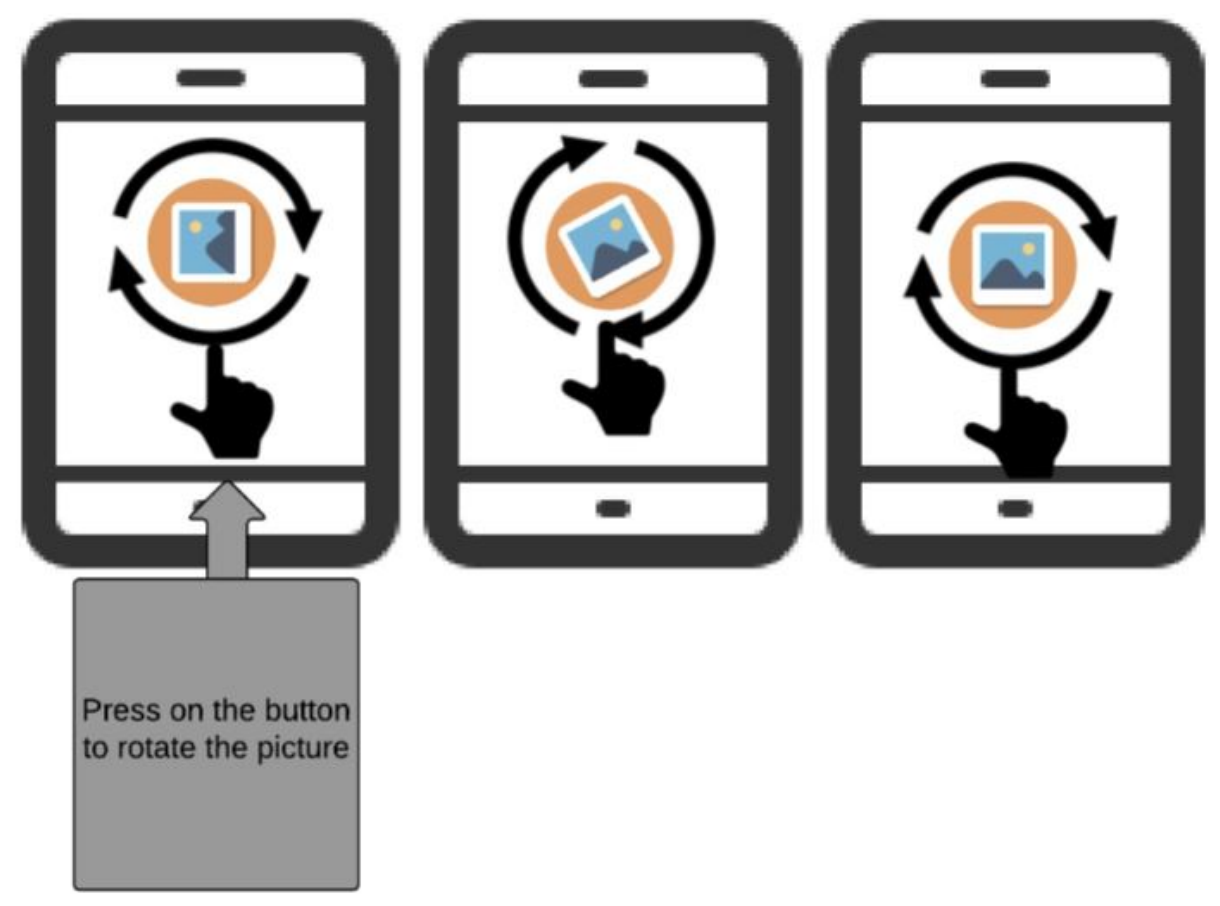

Fig. 2. Task 2 Force-to-rotate

\subsection{Participant Recruitment}

The study was originally designed for older adults, so our aim was to recruit participants above 60 years of age, who used smartphones. The age of above 60 years was chosen as this is broadly in line with some other studies which have taken place in recent years. For example, the study by Findlater et al [6] had an age group of 61-86 years for older users. However, the study by Gao and Sun [15], categorized older users as being between the ages of 52-81.

Finding a diverse group of older adults was the aim for this evaluation. Snowball sampling [16] was used to aid participant recruitment, as well as placing written information with contact details in certain community halls.

Whilst it was difficult to recruit a large sample of participants, nine volunteers were recruited - six male and three female. The youngest participant was 61 years old, and the oldest 82 years old. The average age was 69 (68.88 years average) years. Each participant was given an information sheet concerning the research and an ethics consent statement which each participant signed. The information that was collected from participants did not have any identifying information that could be traced back to a particular participant. Table 1 displays some basic background information about the participants. 
Table 1. Participants' Background Information

\begin{tabular}{|c|c|c|c|}
\hline Participant & Age & Gender & $\begin{array}{c}\text { Current Mobile } \\
\text { Phone }\end{array}$ \\
\hline P1 & 62 & Male & Android \\
\hline P2 & 65 & Male & Apple \\
\hline P3 & 61 & Male & Nokia \\
\hline P4 & 71 & Female & Android \\
\hline P5 & 82 & Male & Apple \\
\hline P6 & 77 & Female & Apple \\
\hline P7 & 62 & Female & Apple \\
\hline P8 & 69 & Male & Apple \\
\hline P9 & 71 & Male & Apple \\
\hline
\end{tabular}

To make the participants comfortable, it was decided to meet them personally at their preferred locations, such as cafés or in their homes. This helped us get enough time from the participants to conduct the evaluation appropriately.

Since all participants did not have skills in using these force touch gestures, a training session was conducted to allow the participants to become familiar with the new gestures before beginning the evaluation. In the training session participants were informed about how the interface works. There was no time limit on the training session, and the participants were allowed the time they needed to interact with the force gestures. In practice all participants completed their training in 30 - 45 minutes. Linked to this, a brief written manual was given to the participants. This detailed how to operate force touch gestures. The aim of using a manual was to try and ensure that all participants had a similar level and understanding of force touch gestures. The manual also aimed to help participants to understand the prototype's functionalities and tasks that would follow in the actual evaluation.

\subsection{Conducting the Evaluation}

Once the preparatory aspects described above were concluded, the actual evaluation with the participants, smartphone, designed tasks and interview questions was carried out.

For the interview parts, semi-structured interviews were chosen as they allowed more flexibility than fully structured interviews. However, semi-structured interviews also require more skill from the interviewer and can incur certain difficulties with interpreting the responses of participants [16].

The interview aimed to investigate how participants experienced the interaction with a prototype with force touch interaction.

The evaluation and interviews were done in three consecutive stages.

In the first stage all participants were asked the following initial set of questions:

- Do you use a mobile as part of your daily routine?

- How long have you been using touch screen mobiles? 
- Are you familiar with touch gestures? Do you perform touch gestures like swipe, pinch, and zoom?

- Which device do you use for Reading newspapers?

- How much time do you spend on a mobile daily?

- I would like to begin by getting a sense of what smartphones mean to you.

As the above questions were completed, the second stage consisted of each participant carrying out all four tasks (described above) using the smartphone. The tasks were timed and to achieve the timing the smartphone's screen was recorded using UX Recorder [17].

Participants were also encouraged to think aloud with their general thoughts as they carried out the four tasks. This was to try and capture fleeting opinions as the interaction proceeded.

When the four tasks were completed, the evaluation moved to the third stage. The issues in the following set of questions and topic areas were covered with each participant:

- In your force touch experience, have you had any difficulties while performing the tasks?

- Which task was easy to perform and which one hard?

- Do you think the newly designed gestures help you to increase font size and image size easily compared to your old conventional methods?

- Are the newly designed gestures easy to learn?

- Did you find any difficulty in performing force touch on the screen?

- Did you manage to use force touch gestures?

- Did you feel that you could solve your tasks easily and without help from others?

- Improvements/Suggestions - Do you have any general suggestions that could make these gestures easier to use?

During the concluding part of the interview, participants were presented with a list composed of simple and complex touch gestures to ask them about simplicity aspects and the possible commands that could be created based on force touch-based gestures. The list the participants were asked to look at was:

- Force to Zoom Image,

- Two Finger Pinched to Zoom Image,

- Two Finger Pinched to Enlarge the text Size,

- Force to Enlarge Text Size,

- Force to rotate Image,

- Two Finger touch to rotate to move them clock and anti-clock wise direction,

- Force Touch to Pop up new window,

- Two finger Press and Tap,

- Flick: Scroll Quickly,

- Force touch to scroll. 


\section{$4 \quad$ Results}

A summary of the results of the three stages described in the previous section will now follow.

For the first stage, all participants declared the following:

- A smartphone was used as part of their daily routine.

- Smartphone usage experience ranged from 1-2 years to 6 years.

- Eight out of nine participants had some familiarity with touch gestures such as swipe, pinch and zoom.

- Three out of nine participants did some reading of newspapers on an electronic device. Several participants mentioned that the text was too small for reading on an electronic device.

- Participants spent between 3-7 hours per day using their smartphone.

- Participants' smartphones were used for a variety of tasks. The most frequent activities were making calls along with aspects of taking and sharing photographs. Other activities that were mentioned included socialising, social media use, entertainment, calendar use, listening to music, setting alarms and checking the time.

For the second stage, times were recorded for each task that was carried out. All nine participants were able to complete the tasks to the required standard. Quite low standard deviations suggest consistency in the data. Table 2 shows the data collected for the task times and nine participants.

Table 2. Force Touch vs. Multi-Touch Performance in Seconds

\begin{tabular}{|c|c|c|c|c|}
\hline & \multicolumn{4}{|c|}{ Time Performance - Seconds (Rounded to Nearest Second) } \\
\hline Participant & $\begin{array}{c}\text { Force-to-Zoom } \\
\text { Text }\end{array}$ & $\begin{array}{c}\text { Pinch-to-Zoom } \\
\text { Text }\end{array}$ & $\begin{array}{c}\text { Force-to-Rotate } \\
\text { Image }\end{array}$ & $\begin{array}{c}\text { Pinch-to-Rotate } \\
\text { Image }\end{array}$ \\
\hline P1 & 6 & 5 & 3 & 12 \\
\hline P2 & 7 & 6 & 4 & 12 \\
\hline P3 & 5 & 8 & 3 & 14 \\
\hline P4 & 10 & 7 & 5 & 15 \\
\hline P5 & 9 & 10 & 9 & 13 \\
\hline P6 & 9 & 7 & 8 & 12 \\
\hline P7 & 7 & 6 & 9 & 11 \\
\hline P8 & 9 & 9 & 7 & 11 \\
\hline P9 & 7 & 6 & 7 & 12.44 \\
\hline Mean & 7.67 & 7.11 & 6.11 & 1.33 \\
\hline $\begin{array}{c}\text { Standard Devia- } \\
\text { tion }\end{array}$ & 1.66 & 1.62 & 2.42 & \\
\hline
\end{tabular}

The data was further tested for normality using Shapiro-Wilk tests. The tests suggest that the data is probably normally distributed: Force to Zoom Text W(9) $=0.922$, $\mathrm{P}=0.405$, Pinch to Zoom Text $\mathrm{W}(9)=0.930, \mathrm{P}=0.481$, Force to Rotate Image W(9) $=0.890, \mathrm{P}=0.200$ and Pinch to Rotate Image $\mathrm{W}(9)=0.874, \mathrm{P}=0.136$. 
The data was also analysed for significance using two related t-tests for the text size increase tasks and image rotation tasks. The two types of tasks were analysed separately as they were done separately by the participants and were not dependent on one another.

Concerning the comparison between the force to zoom text and pinch to zoom text tasks, there is no statistically significant difference between the two in terms of times to complete the tasks $\mathrm{t}(9)=0.958, \mathrm{p}=0.366$ (two tailed).

However, for the force to rotate image and pinch to rotate image tasks, there is a statistically significant difference between the two in terms of times to complete the tasks $t(9)=-5.935, p=0.000348$. The image rotation was statistically significantly faster when carried out using the force to rotate interaction.

During the four tasks participants had been encouraged to think aloud with their general thoughts. However, most participants did not do this as they appeared to concentrate on actually physically doing the tasks. The few comments that were given by participants did not reveal anything of interest to the central issues discussed in this paper.

For the third stage, which occurred after the tasks were carried out by the participants, the remainder of the interview topics were considered with the participants. In summary, the participants' experience in using the force-to-zoom and force-to-rotate features showed the following commonalities:

- Three out of nine participants said they did not have any difficulty in doing the tasks. The other six participants said there were some difficulties with the interaction. These ranged from memory problems, not seeing the point of having a force touch functionality to mentioning that it would be possible to use the features since the user manual and explanations given were helpful (see participant recruitment section above).

- One participant found both tasks easy while two other participants found both tasks difficult. The other six participants felt the image rotation task was easier than the font size increase task. One of the participants who felt both tasks were overall difficult also felt that the image rotation task was the easiest of the two tasks. Some of the participant comments seemed to indicate that the image rotation task was easier because there was a clear button on the screen that needed to be force-pressed, while the font increase task did not have a button on the screen.

- In being asked to compare the new force touch methods with current methods, three participants categorically felt that the new methods were not easier, while three participants felt the new force touch methods were easier. The other participants were less clear in their opinions.

- Regarding ease of learning the new force touch methods, four participants stated the method was not easy to learn. One of the four was less categorically committed to this answer, but was nevertheless tending towards it. The other five participants felt it was easy. Four of these felt they were easy because they saw the manual or were told about it. The ninth participant responded that the picture rotation method was easy, while the font increase method was difficult. 
- The participants were also asked if they had difficulty in performing force touch on the screen. One participant quite categorically stated they had no problems with it. However the other eight participants had some problems with the use of force touch. Some mentioned problems with knowing how much force to apply, not knowing how to apply force and aspects of controllability once the force touch was being applied on the screen. Further, one participant stated that rotating (using force-to-rotate) the image was easier, while another stated that it was difficult to stop the picture rotating once it had started rotating.

- Concerning whether participants felt they could solve their tasks without help from others, five participants felt that they could not do so. Some of the main reasons stated were that they could not remember how to do so, they were bad at learning and several stated they needed to get help from someone. The other four participants were more confident in being able to solve their tasks without help from someone. Some stated that they were good at learning, with some days' practice they could do it or they could do it with the help from the user manual.

- The final question asked the participants if they had any suggestions for improvement of these force touch interactions. Some participants did not answer this question with concrete suggestions. However, some suggested that the force touch interaction was a good idea, while some suggested it was not. Some related suggestions were to have a larger button, to use good fonts for discoverability, to enable better control for image rotation and to have a user manual and training. One participant also reinforced the notion that the rotation task (force-to-rotate) was better than the font size increase (force-to-zoom) task.

- Finally, concerning the list of touch gestures that was shown to the participants, the responses suggested that participants found most of the force touch gestures to be simple as compared to the alternative way of doing the same tasks with multi-touch gestures. Also, participants found most of the multi-touch gestures difficult and wanted force touch gestures as an alternative way to perform tasks.

\section{Discussion and Conclusions}

This preliminary investigation has focused mostly on qualitative aspects with some quantitative aspects included. We achieved a reasonable representative sample of 'elderly' users that used smartphones on a daily basis and for some time. The first stage of interviewing revealed that the sample used smartphones for various activities that are not dissimilar to the activities of the rest of a population. It is therefore suggested that these results have validity in terms of having good links with real-life users. For improvement, it would be good to follow up this investigation with a similar one using a larger sample.

The central issue under initial investigation is whether force touch interaction is a usable mechanism for elderly users in a mobile context. In this study, usable primarily concerned task speed and user perceptions. The significance testing indicated that force to zoom was not faster than pinch to zoom in the text size increase tasks that were designed. However the force to rotate was significantly faster when compared to 
the pinch to rotate interaction for the image rotation tasks. This suggests that force touch is not necessarily better in all cases.

The major findings in this initial study concern the perceptions of the participants. While the results suggest that some participants were positive towards the force touch interaction method they were not familiar with, several of them were not so positive about force touch. Some participants stated quite clearly that the new methods were not easy to use and were not easy to remember how to use them. Also, a majority of participants felt that they would need help from someone or a user manual to use these interaction options. These are usability aspects that cannot be ignored. This poses a serious problem when a good part of the participant sample expresses opinions that something is not easy to use in some way. Often when this happens to users, they will tend to 'forget' about the option and use some other equivalent option, even if perhaps less efficient.

Furthermore, most of the participants had some problems in using force touch. When a user experiences problems with some kind of interaction, they are likely to form a negative opinion of it and perhaps not use it at all. This concerned aspects of the interview that specifically linked with the practical use of the touch gestures during the tasks. However, when shown the list of touch gestures out of the context of the tasks they had just done, participants overall seemed positive towards force touch.

Linking the practical results of this preliminary investigation with the relevant universal design principles [2] mentioned in the Related Literature section above indicate some interesting observations.

Concerning P1 - Equitable Use, from the interviews that were carried out this principle is not fully adhered to. This is because several users from the sample had some problems in using force touch. This is despite the sample being one of relatively experience older users.

Thinking about P2 - Flexibility in Use, we would suggest that since current smartphones with force touch capabilities continue to have other equivalent interaction options that there is some flexibility for users to use a device with their own preferences. However, any functionality that can only be operated by force touch would limit flexibility as it would force everyone to use that interaction method whether they like it or not.

Regarding P3 - Simple and Intuitive Use, the interviews indicated that for some of the participants the force touch operation was not simple and intuitive. Some suggested that doing the tasks were facilitated by the user manual or being told about the method. This suggests that if one feels reliant on a manual or help from someone else, that the particular method of interaction could be better in terms of simplicity and being intuitive to use.

For P4 - Perceptible Information, most of the participants felt that the image rotation task was easier. Some indication for the reason for this judgment on the part of the participants was that some stated that having a clear button on the screen for pressing with force for this task made the whole task easier than the task for the font size increase. The authors suggest that the reason for this is that having a button on the screen gave participants better or more perceptible information indicating more clearly what had to be done (ambient conditions or sensory abilities were not investi- 
gated in this study). This is also given more credence by the timing data. For this task the force touch interaction was statistically significantly faster than the pinch to rotate interaction.

From the preliminary investigation discussed above, the authors suggest that a force touch feature implemented on a smartphone can lack somewhat in usability and in the application of certain universal design principles in the context of older users and increasing font size and rotating images. We would suggest that all mobile platform designers and developers (and all other platforms too) should not stop trying to innovate. However, innovation should not sacrifice in any circumstances the usability and inclusivity aspects of a product or feature of a product. Our preliminary results suggest that currently the force touch feature is not inclusive enough as some of the older participants had some problems with the feature. This is particularly in the context of increasing font size and rotating images. We would therefore suggest caution in other related and different contexts and only fully release products with such features once robust published evaluations are available.

Lastly, we would encourage further work in this area to make the results more robust, e.g. by increasing the sample size and perhaps conducting a rigorous empirical experiment to collect more concrete data on performances. This study was more qualitative in nature and exploratory. It also perhaps could be improved by conducting more in-depth interviews.

\section{References}

[1] Bhalla, M.R. and Bhalla, A.V. Comparative study of various touchscreen technologies. International Journal of Computer Applications, 2010. 6(8): p. 12-18. https://doi.org/10.5120/1097-1433

[2] Story, M.F., Maximizing usability: the principles of universal design. Assistive technology: The Official Journal of RESNA, 1998. 10(1): p. 4-12. https://doi.org/10.1080/ 10400435.1998 .10131955

[3] Apple Inc. Getting Started with 3D Touch, https://developer.apple.com/library/ archive/documentation/UserExperience/Conceptual/Adopting3DTouchOniPhone/. 2016, Accessed March 2018.

[4] Clarkson, E.C., Patel, S.N., Pierce, J.S. and Abowd, G.D. Exploring continuous pressure input for mobile phones. GVU Tech. Report, GIT-GVU-06-20, 2006, Georgia Institute of Technology.

[5] Huber, J., Sheik-Nainar, M. and Matic, N. Force-enabled Touch Input on the Steering Wheel: An Elicitation Study. Adjunct Proceedings of the 9th International ACM Conference on Automotive User Interfaces and Interactive Vehicular Applications. 2017. September 24-27, Oldenburg, Germany, ACM.

[6] Findlater, L., Froehlich, J.E., Fattal, K., Wobbrock, J.O. and Dastyar, T. Age-related differences in performance with touchscreens compared to traditional mouse input. Proceedings of the SIGCHI Conference on Human Factors in Computing Systems, Paris, France, April 27 - May 02, 2013. p. 343-346.

[7] Brewster, S.A. and Hughes, M. Pressure-based text entry for mobile devices. in Proceedings of the 11th International Conference on Human-Computer Interaction with Mobile Devices and Services. MobileHCI09, ACM, September 15 - 18, Bonn, Germany, 2009. 
[8] Hwangbo, H., Yoon, S.H., Jin, B.S., Han, Y.S. and Ji, Y.G. A study of pointing performance of elderly users on smartphones. International Journal of Human-Computer Interaction, 2013. 29(9): p. 604-618. https://doi.org/10.1080/10447318.2012.729996

[9] Kim, I. And Jo, J.H. Performance comparisons between thumb-based and finger-based input on a small touch-screen under realistic variability. International Journal of HumanComputer Interaction, 2015. 31(11): p. 746-760. https://doi.org/10.1080/ 10447318.2015.1045241

[10] Hertzum, M. and Hornbæk, K. How age affects pointing with mouse and touchpad: A comparison of young, adult, and elderly users. Intl. Journal of Human-Computer Interaction, 2010. 26(7): p. 703-734. https://doi.org/10.1080/10447318.2010.487198

[11] Akoumianakis, D. and Stephanidis, C.. Universal design: towards universal access in the information society. in CHI'01 Extended Abstracts on Human Factors in Computing Systems. 2001. ACM.

[12] Yamartino, S. ressure is a JavaScript library for handling Force Touch, 3D Touch and Pointer Pressure on the web. 2016 [cited 2016 01-01-2016]; Available from: https://pressurejs.com/.

[13] Statista. Most Popular Mobile Internet Activities According to Internet Users Worldwide as of 1st Half 2017, By Device, https://www.statista.com/statistics/249761/most-popularactivities-carried-out-on-mobile-internet-devices/, 2018, Accessed March 2018.

[14] Apple Inc. iPhone 6s, https://www.apple.com/lae/iphone-6s/specs/, 2018 Accessed March 2018.

[15] Gao, Q. and Sun, Q. Examining the usability of touch screen gestures for older and younger adults. Human factors, 2015. 57(5): p. 835-863. https://doi.org/10.1177/00187 $\underline{20815581293}$

[16] Lazar, J., Feng, J.H. and Hochheiser, H. Research methods in human-computer interaction. 2017: Morgan Kaufmann.

[17] Foraker Labs. UX Recorder, http://www.uxrecorder.com/, 2018, Accessed April 2018.

\section{$7 \quad$ Authors}

Dr Pietro Murano is an Associate Professor of Computer Science. OsloMet Oslo Metropolitan University, Department of Computer Science, Universal Design of ICT Research Group, PO box 4, St. Olavs plass, 0130 Oslo, Norway. http://www.pietromurano.org

Rao Muhammad Danial Ali is a Master of Universal Design of ICT. OsloMet Oslo Metropolitan University, Department of Computer Science, Universal Design of ICT Research Group, PO box 4, St. Olavs plass, 0130 Oslo, Norway.

Article submitted 10 July 2018. Final acceptance 15 August 2018. Final version published as submitted by the authors. 